\title{
PERSPECTIVAS ESTUDIANTILES SOBRE ACTIVIDADES DE INSTRUCCIÓN ENTRE PARES DESARROLLADAS EN UN CURSO DE MECÁNICA INTRODUCTORIA
}

\section{Students' perspectives on peer instruction activities developed in an introductory mechanics course}

DOI https://doi.org/10.36411/AEF.1.2.9

Nicolás Budini

ORCID https://orcid.org/0000-0003-3059-4703.

Facultad de IngenieríaQuímica, Universidad Nacional del Litoral, Santa Fe, Argentina. Instituto de Física del Litoral (UNL-CONICET), Santa Fe, Argentina.

Luis Marino

ORCID https://orcid.org/0000-0003-3574-3106

Facultad de Humanidades y Ciencias, Universidad Nacional del Litoral, Santa Fe, Argentina

Sandra Gómez Carrillo

ORCID https://orcid.org/0000-0002-3246-2803

Facultad de Ingeniería Química, Universidad Nacional del Litoral, Santa Fe, Argentina

Ricardo Carreri

ORCID https://orcid.org/0000-0001-5570-2454

Facultad de Ingeniería Química, Universidad Nacional del Litoral, Santa Fe, Argentina

Cristina Cámara

ORCID https://orcid.org/0000-0002-1423-9931

Facultad de IngenieríaQuímica, Universidad Nacional del Litoral, Santa Fe, Argentina Facultad de Ciencias Agrarias, Universidad Nacional del Litoral, Santa Fe, Argentina.

Silvia Giorgi

https://orcid.org/0000-0002-8730-8862

Facultad de IngenieríaQuímica, Universidad Nacional del Litoral, Santa Fe, Argentina. giorgi.silvia@gmail.com 


\title{
Resumen
}

En el marco de un proyecto de investigación en educación en Física se están estudiando los beneficios, para el aprendizaje, derivados de la implementación de la modalidad didáctica denominada Instrucción entre Pares en un curso introductorio de Mecánica de nivel universitario. En el contexto de esta metodología de aprendizaje activo, los conceptos y relaciones se abordan a través de preguntas conceptuales, cuyas respuestas involucran instancias de aprendizaje colaborativo. En este trabajo presentamos los resultados obtenidos a partir de una encuesta diseñada para evaluar las perspectivas de estudiantes que ya habían terminado un primer curso de Mecánica en el que se implementó dicha metodología. Las valoraciones positivas de ellos con respecto a las clases en las que se aplicó la Instrucción entre Pares nos alientan a continuar implementándola.

Palabras claves: Mecánica introductoria. Instrucción entre Pares. Estudiantes. Perspectivas.

\begin{abstract}
Within the framework of a research project in physics education we are studying the benefits, for learning, derived from the application of a didactic modality called Peer Instruction in an introductory course of Mechanics at the university level. In the context of this active learning methodology, concepts and relationships are addressed through conceptual questions, whose answers involve instances of collaborative learning. In this paper, we present the results obtained from a survey designed to evaluate the perspectives of students who have already completed a first Mechanics course in which this methodology was applied. Their positive assessments regarding the classes where Peer Instruction was applied encourage us to continue using this methodology.
\end{abstract}

Keywords: Introductory Mechanics. Peer Instruction. Students. Perspectives.

\section{Introducción}

Las dificultades de los estudiantes del ciclo inicial universitario en el aprendizaje de los conceptos que se desarrollan en un curso introductorio de Mecánica son generalizadas. Esto se ve reflejado en los bajos rendimientos observados en las diversas instancias de evaluación. Estas dificultades inherentes a los estudiantes en cuanto a la comprensión de los conceptos involucrados en los cursos iniciales de física han sido evidenciadas sistemáticamente y globalmente a partir de los resultados obtenidos por la investigación en enseñanza de la física durante las últimas décadas (Byun y Lee, 2014).

Con la intención de mejorar la comprensión conceptual de los estudiantes respecto de los contenidos de Física, Eric Mazur (1997) desarrolló un método de enseñanza interactivo denominado instrucción entre pares (IP), que implementó exitosamente en universidades estadounidenses. Eric Mazur (op. cit.) señala, entre las ventajas de dicha modalidad, que puede ser fácilmente adaptada y aplicada en otros contextos de enseñanza.

Durante sus clases, Mazur (op. cit.) encontró que muchos de sus estudiantes son en general muy buenos para resolver problemas de tipo cuantitativos, es decir cuyos resultados esperados son de tipo numéricos, pero los mismos estudiantes no responden adecuadamente a preguntas conceptuales en las que tienen que elaborar respuestas cualitativas simples en base a esos mismos conceptos. Mazur indica que de este hecho se derivan cuestionamientos no sólo sobre los criterios o métodos de evaluación, ya que un estudiante puede aprobar un examen sin haber comprendido realmente los conceptos necesarios, sino también sobre la formación que se busca con el cursado de una asignatura.

Una comprensión genuina de los conceptos y sus relaciones conduce incuestionablemente, a una mejor formación de los estudiantes no sólo para abordar problemas cuantitativos sino también cualitativos. 
El hecho de que los estudiantes sepan cómo enunciar una ley física o cómo reemplazar variables por números en una ecuación no debería constituir un resultado relevante esperado de la enseñanza. La actividad docente debería estar regida por la responsabilidad de aspirar a enseñar, de manera que se logre una formación más sólida y eficiente de los estudiantes. Para esto, hoy en día, existen diversas herramientas y estrategias didácticas ampliamente probadas en diversos contextos a nivel mundial que son efectivas en cuanto a promover el interés de los estudiantes por aprender y a mejorar el nivel conceptual de los mismos (Nicol y Boyle, 2003; Cortrightet al., 2005; Freeman et al., 2014; Giuliodoriet al., 2006; Martín del Pozo et al., 2017; Navío-Marco y Solórzano-García, 2019). Dos revisiones interesantes de diferentes estrategias de enseñanza de la física y sus fundamentos pueden encontrarse en Redish (2003) y Meltzer y Thornton (2012).

La aplicación de la IP de la que derivan los resultados aquí presentados busca fortalecer el aprendizaje conceptual de los contenidos de Mecánica por parte de los estudiantes, complementando a las otras instancias de aprendizaje tradicionales del ámbito universitario. Las actividades se están llevando a cabo en la asignatura Física I, del ciclo inicial de las carreras que se cursan en la Facultad de Ingeniería Química (FIQ) de la Universidad Nacional del Litoral (UNL), en el marco de un proyecto de investigación que busca evaluar los alcances de la IP en la comprensión de los contenidos por parte de los estudiantes.

Esta asignatura es de cursado común para las carreras: Ingenierías en Alimentos, Industrial, en Materiales y Química, Licenciaturas en Física, Matemática Aplicada, Materiales y Química, y Profesorado en Química. Los estudiantes se encuentran habilitados a cursar Física I habiendo aprobado un curso básico de matemática y regularizado un curso de cálculo diferencial e integral.

En los planes de estudios del ciclo inicial de las carreras mencionadas, Física I está programada para ser cursada durante el primer cuatrimestre del segundo año lectivo. Es un curso de 120 horas totales de duración que se distribuyen en 8 horas semanales de cursado durante las 15 semanas de duración de un cuatrimestre. Estas horas semanales se distribuyen en las siguientes actividades: clases tradicionales de teoría de 2,5 horas (obligatorias), clases complementarias de teoría (CC) de 2 horas (opcionales), clases tradicionales de trabajos prácticos de laboratorio de 3 horas (obligatorias), clases tradicionales de resolución de problemas de 2,5 horas (opcionales). La bibliografía recomendada se basa en los libros de Mecánica tradicionales con cálculo, los estudiantes también disponen de un Apunte de Cátedra en el que se desarrollan los contenidos con la rigurosidad conceptual y matemática que se espera que manejen los estudiantes.

El número de estudiantes que cursa la asignatura por cuatrimestre es variable (entre 100 y 140 estudiantes). Las clases de teoría tradicionales, así como también las CC se dictan para todo el alumnado en conjunto, se puede encontrar una descripción detallada de estas últimas en los trabajos de Budini; Giorgi; Sarmiento; Cámara; Carreri; Marino\&Gómez Carrillo1 (2016);Budini; Cámara; Sarmiento; Cámara\&Carreri (2017) yBudini; Marino, Carreri; Cámara\&Giorgi (2018). De todos los estudiantes que cursan Física I asisten a las CC los estudiantes a los que no se les superponen horarios con actividades obligatorias de otras asignaturas que se cursan en paralelo con Física I, esto es un $80 \%$ del total de estudiantes. A principios de cuatrimestre prácticamente todos los estudiantes asisten a las CC, a medida que transcurren las semanas el número de asistentes varía según las 
demandas de las otras asignaturas de cursado paralelo, fundamentalmente disminuye en vísperas de exámenes parciales.

Caber mencionar que el docente a cargo de dictar las clases tradicionales de teoría no es el mismo que el que desarrolla las CC. Estas clases se incluyeron en el cursado de Física I con el objetivo de implementar la IP en el marco del proyecto de investigación en enseñanza y aprendizaje de la Física mencionado anteriormente.

Otra cuestión que merece ser mencionada es que en las CC sólo se dan las condiciones para que la IP pueda ser aplicada adecuadamente durante el desarrollo de la primera parte de los contenidos de la asignatura (Cinemática de partículas hasta Dinámica y Energía de sistemas físicos no puntuales), esto se debe a que para el desarrollo de los temas siguientes (Movimientos oscilatorios, Ondas, Fluido estática y Fluidodinámica) el tiempo de cursado se torna escaso, por lo que las CC se destinan a reforzarlos a través de consultas, resolución de problemas de exámenes, etc.

En este trabajo se analizan y comunican los resultados derivados de una encuesta de opinión generada ad hoc y contestada por estudiantes que se encuentran cursando Física II, lo cual implica que ya cursaron de Física I y que participaron de las clases complementarias (CC) en las que se implementó la IP durante 2017. Se describen las perspectivas y valoraciones de 29 estudiantes acerca de determinados aspectos de la aplicación de la IP. Algunos de estos estudiantes ya habían aprobado la asignatura Física I por exámenes de término medio (parciales) durante el cursado, otros habían aprobado por examen final luego de finalizar el curso y la fracción restante aún no la habían aprobado.

\section{Marco teórico y objetivos}

Con la enseñanza de la Física, a través de las distintas instancias de aprendizaje, se busca favorecer en los estudiantes la reconstrucción de un cuerpo claro, estable y organizado de conocimientos con el que puedan resolver diversas situaciones y a la vez adquirir conocimientos que demandan un mayor nivel de abstracción dentro del mismo campo, o afines al mismo. Para lograr estos objetivos, un importante número de resultados derivados de la investigación en enseñanza muestran la necesidad de que los estudiantes participen activamente en su proceso de aprendizaje, en un contexto de enseñanza que propicie la reconstrucción conceptual, es decir que propicie al aprendizaje considerado como procesos en los que los alumnos desarrollan sus estructuras mentales formando nuevas concepciones basadas en su propia imaginación y experiencia (Vigotsky, 1989; Ausubel, Novak\&Hanesian, 1991; Duit, Gropengießer, Kattmann, Komorek\&Parchmann, 2012).

El aprendizaje colaborativo (AC), brinda un marco propicio para reconstruir conocimientos. El mismo se basa en concebir a la educación como un proceso de socio-construcción (Vigostky, op. cit.). El AC es una modalidad didáctica que busca mejorar la comprensión del estudiante sobre un tema a partir del trabajo en pequeños grupos con diferentes niveles de habilidad. Así, los estudiantes aprenden activamente en un ambiente más relajado y flexible, en clases más dinámicas que las tradicionales. Calzadilla (2002) señala que el AC conduce a los estudiantes al logro mutuo de un nuevo nivel de conocimiento y satisfacción. En 
ese sentido se señala que una de las etapas cruciales en la implementación de la IP se constituye en una instancia en la que se pone en juego ese tipo de aprendizaje.

En el marco de una investigación que estudia los beneficios de la IP en la enseñanza y el aprendizaje de contenidos de Mecánica, dicha modalidad se implementa durante el cursado de Física I en la FIQ-UNL, durante las mencionadas CC con periodicidad semanal y de carácter no obligatorio. Estas clases, de alrededor de dos horas de duración, se dictan luego de que los estudiantes asistieron a la clase teórica tradicional en la que se introduce y desarrolla el tema a abordar y de que leyeron un breve material escrito (notas de clase) del que disponen previamente. Es fundamental en las clases sustentadas en la IP la necesidad de que los estudiantes hayan sido introducidos previamente al tema, de manera de enfocar la atención en los conceptos físicos básicos, o en aquellos que ofrezcan mayores dificultades de comprensión. El diseño de estas clases requiere tener en cuenta los siguientes puntos claves detallados en Budini et al., 2016, p. 187;Budini; et al., 2017, p. 287;Budini et al., 2018, p. 142):

(1) identificar las cuestiones que más dificultades presentan a los estudiantes y elegir unos pocos conceptos físicos involucrados en las mismas para reforzar en las CC;

(2) diseñar actividades interactivas alrededor de esos pocos conceptos que resulten motivadoras,

(3) formular preguntas conceptuales (Mazur, 1997) alrededor de los conceptos que se desean reforzar, previamente seleccionadas, para que los estudiantes respondan en el momento.

Luego de un breve repaso de los conceptos centrales se implementa la IP presentando a los estudiantes las denominadas preguntas conceptuales (PC) acerca de los conceptos y relaciones que se intentan reforzar. Las mismas son del tipo de opción múltiple de respuestas, de las que sólo una es la correcta. Luego de que los estudiantes responden una PC reciben inmediatamente la retroalimentación por parte del profesor. En esa instancia se discute con toda la clase por qué una opción de respuesta es la correcta y por qué las otras no lo son. De esta manera, los estudiantes pueden reflexionar para aclarar los conceptos en el momento y no esperar a la próxima clase, lo cual genera en ellos motivación para comprender el tema.

En los trabajos de Budini et al. $(2016 ; 2017 ; 2018)$ se pueden encontrar descripciones de las actividades desarrolladas en las CC. Lo más relevante para remarcar en este trabajo es que la modalidad IP se basa en gran medida en la calidad y relevancia de las PC. Las mismas pueden encontrarse y seleccionarse del libro de Mazur (1997) o diseñarse teniendo en cuenta los siguientes criterios básicos (Beatty et al., 2006):

a) deben involucrar pocos conceptos y centrarse en uno solo,

b) sus respuestas no deben desprenderse del reemplazo de valores numéricos en ecuaciones,

c) las opciones de respuestas (opción múltiple) deben ser suficientes, y de ser posible involucrar las concepciones no científicas más comunes de los estudiantes para que salgan a la luz en la discusión final con el docente, 
d) deben estar redactadas de forma inequívoca,

e) deben ser de dificultad moderada, según el contexto en el que se están formulando.

La manera de trabajar las PC en las CC de Física I en la FIQ (Budini et al., 2016 ; 2017; 2018) consiste en las siguientes etapas:

(1) el/la docente, muestra la pregunta y las opciones posibles de respuestas, en una pantalla haciendo uso de un proyector, y las lee en voz alta para asegurarse de que no haya confusiones sobre el enunciado,

(2) los/as estudiantes, utilizando sus smartphones, acceden a la pregunta y a las opciones de respuestas a través de un formulario web,

(3) se otorgan alrededor de dos minutos para que, individualmente, cada estudiante seleccione la respuesta que cree correcta y la registre en el formulario web junto con el nivel de confianza (muy seguro, todavía pensando y poco seguro),

(4) luego, los estudiantes discuten con sus compañeros/as ubicados/as cercanamente (discusión entre pares, propiamente dicha), durante alrededor de cinco minutos, acerca de qué opción seleccionaron y por qué; en esta instancia son ellos/as mismos/as quienes elaboran los argumentos que usaron para seleccionar tal o cual opción,

(5) posteriormente, los/as estudiantes vuelven a registrar individualmente las respuestas y niveles de confianza en el formulario; quienes hayan cambiado de idea al terminar la discusión pueden modificar su respuesta y nivel de confianza,

(6) finalmente, las proporciones de respuestas y niveles de confianza antes y después de la discusión se muestran a todo el grupo y se discute de manera global, por un lado, cuál es la respuesta correcta y qué lo fundamenta, y por otro, por qué las otras opciones no son correctas.

Luego de la primera elección de respuesta y nivel de confianza por parte de cada estudiante, la discusión de ellos/as con sus pares promueve su participación activa y su pensamiento basado en los argumentos que se están desarrollando en grupos (AC). Esto les brinda a ellos, así como al profesor, una forma de evaluar el grado de comprensión que han logrado. Las discusiones entre los estudiantes promueven algo más importante aún, ellos no se limitan a asimilar el material que se les presenta, ya que deben reflexionar y poner sus pensamientos en palabras. A veces los mismos estudiantes son capaces de explicar un concepto a sus compañeros con más eficacia que el docente, probablemente porque ellos, habiendo comprendido el concepto recientemente, son conscientes de las dificultades que conlleva y saben exactamente qué enfatizar en su explicación.

Las ventajas del uso de formularios web son importantes en dos sentidos: se tiene un panorama inmediato de la distribución de respuestas y niveles de confianza que da lugar a que los estudiantes cuenten con retroalimentación en el momento, esto permite cuantificar la eficacia de la interacción entre estudiantes para que ellos analicen y discutan sus respuestas; es importante también para el docente que queden registros de las opciones elegidas por los estudiantes antes y después de las discusiones entre ellos, de manera de contar con la posibilidad de un análisis más profundo de la información que permitiría conocer los aprendizajes alcanzados. 
En este trabajo se presentan resultados derivados de las respuestas a una encuesta de opinión sobre aspectos de las PC y la manera de abordarlas en las CC, y sobre la forma de trabajar en dichas clases, contestada por estudiantes que han participado en las mismas y que se encuentran en distintas situaciones respecto a la aprobación de la asignatura, algunos la aprobaron a través de exámenes de término medio, otros a través de examen final y otros no la aprobaron aún.

\section{Metodología}

Se aplicó una encuesta ad hoc a 29 estudiantes que se encontraban cursando la asignatura Física II y que habían asistido a las CC durante el cursado previo de Física I. Cabe aclarar que los estudiantes se encuentran habilitados a cursar Física II habiendo, como mínimo, regularizado Física I a través de haber cumplido con:

- El $80 \%$ de asistencia a las clases de teoría tradicionales.

- La aprobación de cinco evaluaciones realizadas durante las quince semanas de cursado, una por cada unidad temática: Errores de mediciones, Cinemática, Dinámica y Energía de partículas, Dinámica y Energía de sistemas de partículas, Oscilaciones y Ondas. Se exceptúa en estas evaluaciones los temas Fluido Estática y Fluido Dinámica por razones de tiempo.

- La asistencia y aprobación de todos los trabajos prácticos de laboratorio (11 en total) y sus respectivos informes.

El propósito de una parte de la encuesta fue indagar, por un lado, sobre las valoraciones de los estudiantes respecto a ciertos aspectos de las PC y a la manera de trabajar dichas preguntas en las CC y, por otro, conocer las opiniones de los mismos sobre los beneficios que consideraban alcanzados debido a su participación en las CC.

La encuesta utilizada como instrumento de recolección de información para la evaluación de la implementación de la IP en Física I fue diseñada en forma pre estructurada (Sampieri, Collado \& Lucio, 2010) y contestada por los estudiantes de manera escrita. En el Apéndice se muestran las consignas, cuyas respuestas se analizan y discuten en este trabajo.

\section{Resultados y discusión}

En el trabajo de Budini et al. (2018) se caracterizó a la muestra de estudiantes que cursó Física I en el año 2017 en relación a su situación respecto a la promoción de Física I. Se informó que el 24\% (7/29) de ellos había aprobado la asignatura por exámenes de término medio (o exámenes parciales), el 45\% (13/29) por examen final y el 31\% (9/29) aún no la había aprobado. También se informaron parte de los resultados obtenidos a partir de las respuestas de los estudiantes al cuestionario escrito mencionado. En esta oportunidad se intenta avanzar en el análisis de otros resultados derivados de los ítems del cuestionario que se muestran en el Apéndice.

Una consigna solicitó las valoraciones de los estudiantes respecto a aspectos de las PC y a la manera en que se abordan(Figura 1). Las opciones para poder ex- 
presar dichas valoraciones eran: Muy útil, Útil y Poco útil. Concretamente, los estudiantes debían valorar los siguientes aspectos relativos a las PC y a la manera de abordarlas en las CC:

El planteo de preguntas cuyas respuestas involucran relaciones entre conceptos. El requerimiento de diversos análisis a partir de las respuestas de opción múltiple a las preguntas, en el sentido que luego de responder cada PC el docente discute con toda la clase acerca de la opción de respuesta correcta y por qué las otras opciones son incorrectas.

La interacción de él/ella con sus compañeros/as antes de la segunda respuesta. Su interacción con el docente al analizar las respuestas.

A partir de los resultados presentados se puede decir que lo más destacable es que, independientemente de su situación respecto a la aprobación de la asignatura, los estudiantes opinan que les resultó de mucha utilidad el planteo de preguntas cuyas respuestas involucran relaciones entre conceptos y la posibilidad de interacción con el docente para analizarlas.

Particularmente, a más de la mitad de los estudiantes que aprobaron Física I a través de examen final, también les resultó muy útil el requerimiento de diversos análisis a partir de las respuestas de opción múltiple a las preguntas.

Por su parte, los estudiantes que no habían aprobado Física I aún, también valoraron como útiles el requerimiento de diversos análisis a partir de las respuestas de opción múltiple a las preguntas y además la interacción con sus compañeros/as antes de la segunda respuesta.

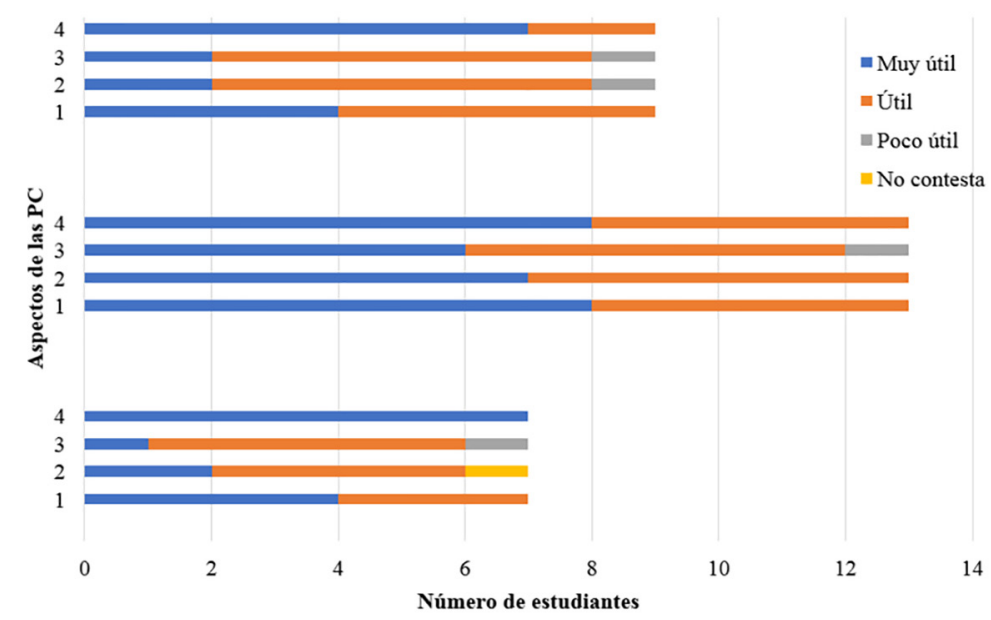

Figura 1. Valoraciones por parte de los estudiantes de aspectos de las PC y de la manera de abordarlas en las CC, y su situación relativa a la aprobación de Física I.

1. El planteo de preguntas cuyas respuestas involucran relaciones entre conceptos. 2. El requerimiento de diversos análisis a partir de las respuestas de opción múltiple a las preguntas. 3- La interacción de él/ella con sus compañeros/as antes de la segunda respuesta. 4- Su interacción con el docente al analizar las respuestas.

Se estudiaron también las opiniones sobre diferentes afirmaciones acerca del contexto en el que se trabajan las preguntas conceptuales en las CC. Las opciones, presentadas para opinar en relación a las diferentes afirmaciones eran: Totalmente en desacuerdo; Parcialmente en acuerdo; Totalmente en acuerdo. Las afirmaciones se muestran a continuación: 
- La forma de abordar las preguntas en las clases propició un ambiente de trabajo más agradable para aprender, que en otros tipos de clases.

- Tu participación online, a través de la herramienta del Google doc., hizo que te sientas más activo/a durante la clase.

- Durante el desarrollo de la clase te sentiste cómodo/a al interactuar con sus compañeros/as.

- Resulta formativo trabajar, discutir y razonar en conjunto con compañeros/as.

- La metodología te sirvió para clarificar y afianzar conocimientos desarrollados en las clases de teoría.

En la Figura 2, se muestran los resultados hallados, discriminados en base a la situación de los estudiantes con respecto a la promoción de la asignatura.

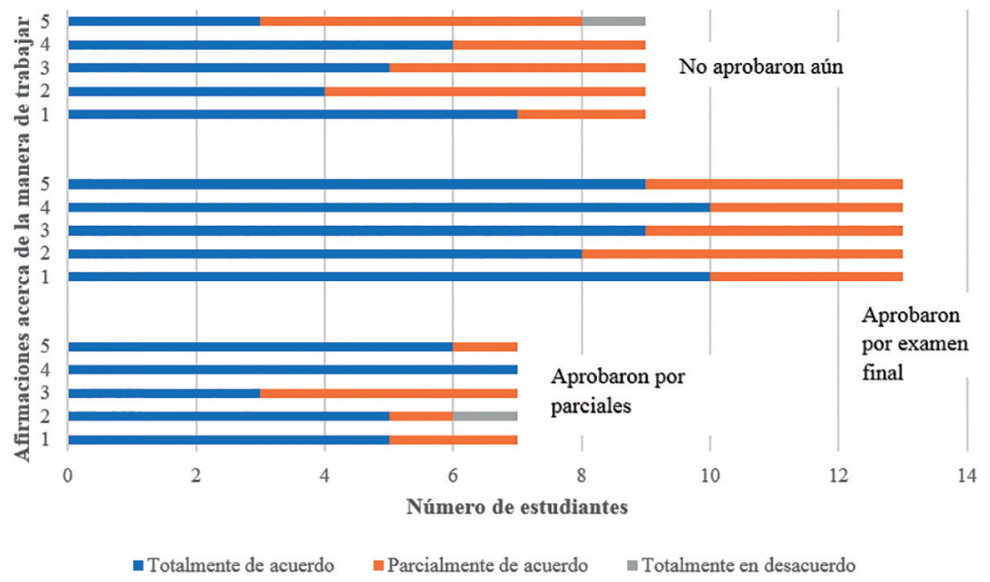

1- La forma de abordar las preguntas en las clases propició un ambiente de trabajo más agradable para aprender, que en otros tipos de clases. 2- Tu participación online, a través de la herramienta del Google doc., hizo que te sientas más activo/a durante la clase. 3- Durante el desarrollo de la clase te sentiste cómodo/a al interactuar con sus compañeros/as. 4- Resulta formativo trabajar, discutir y razonar en conjunto con compañeros/as. 5- La metodología te sirvió para clarificar y afianzar conocimientos desarrollados en las clases de teoría.

Figura 2. Opiniones de los estudiantes sobre las afirmaciones acerca de la manera de trabajar en las CC y su situación relativa a la promoción de la materia.

Del análisis de la misma se desprende que más de la mitad de los estudiantes que aprobaron por parciales contestaron que estaban totalmente de acuerdo con las afirmaciones relacionadas con que resulta formativo trabajar, discutir y razonar en conjunto con compañeros/as en primer lugar, con que la metodología les sirvió para clarificar y afianzar conocimientos desarrollados en las clases de teoría en segundo lugar, con que la forma de abordar las preguntas en las clases propició un ambiente de trabajo más agradable para aprender, que en otros tipos de clases en tercer lugar, y con que la participación online, a través de la herramienta del Google doc., hizo que se sientan más activos/as durante la clase en cuarto lugar.

La mayoría de los estudiantes que aprobó a través de examen final acordaron con las afirmaciones relacionadas con que la forma de abordar las preguntas en las clases propició un ambiente de trabajo más agradable para aprender, que en otros tipos de clases en primer lugar, con que resulta formativo trabajar, discutir y razonar en conjunto con compañeros/as en segundo lugar, con que durante 
el desarrollo de la clase se sintieron cómodos/as al interactuar con sus compañeros/as en tercer lugar, con que la metodología les sirvió para clarificar y afianzar conocimientos desarrollados en las clases de teoría en cuarto lugar, y en quinto lugar con que la participación online, a través de la herramienta del Google doc., hizo que se sientan más activos/as durante la clase.

Con respecto a los estudiantes que aún no habían aprobado Física I, valoraron positivamente a la forma de abordar las preguntas en las clases en el sentido que propició un ambiente de trabajo más agradable para aprender, que en otros tipos de clases en primer lugar, a que resulta formativo trabajar, discutir y razonar en conjunto con compañeros/as en segundo lugar, a que durante el desarrollo de la clase se sintieron más cómodos/as al interactuar con sus compañeros/ as en tercer lugar, a que la participación online, a través de la herramienta del Google doc., hizo que se sientan más activos/as durante la clase en cuarto lugar, y en quinto lugar a que la metodología les sirvió para clarificar y afianzar conocimientos desarrollados en las clases de teoría.

Con relación a las opiniones de los estudiantes sobre los beneficios alcanzados debido a su participación en las CC, interesó conocer si las mismas les resultaron útiles para aprender a razonar, o analizar las situaciones problemáticas. Los resultados obtenidos se muestran en la Figura 3.

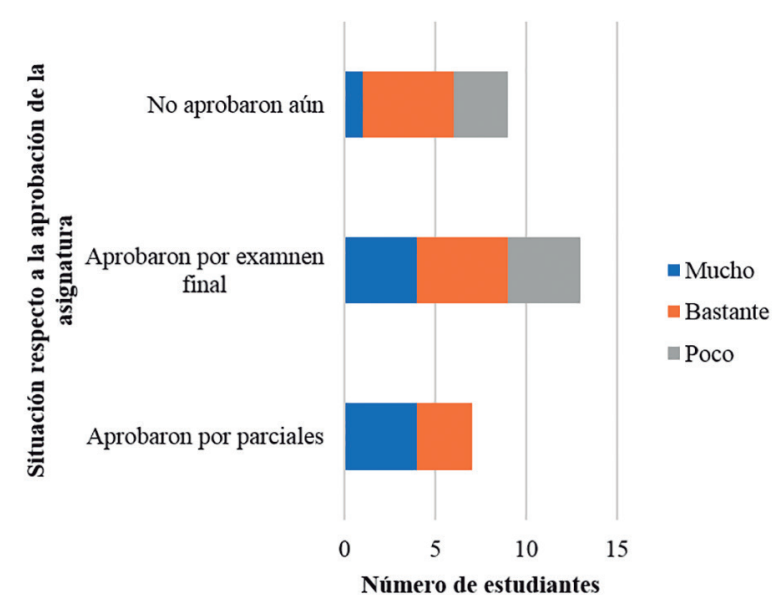

Figura 3.Valoraciones sobre el aporte de las actividades desarrolladas en las CC en la manera de razonar o analizar situaciones problemáticas.

De los estudiantes que promocionaron Física I por examen final, la proporción más alta consideró a las CC bastante útiles, y similares proporciones las consideraron muy, y poco, útiles. Los alumnos que aún no habían aprobado Física I, opinaron mayoritariamente que las CC les resultaron bastante útiles y en menor proporción poco útiles. Por su parte, los que habían aprobado por parciales consideraron a las CC exclusivamente muy, y bastante, útiles. 


\section{Conclusiones}

Los resultados mostrados alientan a continuar aplicando la IP durante el cursado de Física I en la FIQ. Con respecto a las valoraciones por parte de los estudiantes sobre características de las PC y de la manera de abordarlas en las CC, se puede decir, que independientemente de su situación respecto a la aprobación de la asignatura, los mismos opinaron que les resultó de mucha utilidad el planteo de preguntas cuyas respuestas involucran relaciones entre conceptos y la posibilidad de interacción inmediata con el docente para analizar las respuestas. También, principalmente los estudiantes que aprobaron Física I a través de examen final, apreciaron en forma positiva el requerimiento de diversos análisis sobre las respuestas presentadas para optar por la que consideraban correcta.

Teniendo en cuenta las opiniones de los estudiantes sobre el contexto en el que se trabajan las PC en las CC, se destaca que todos los estudiantes opinaron mayoritariamente que resulta formativo trabajar, discutir y razonar en grupo, en acuerdo con las bases del AC (Calzadilla, 2002).

Por su parte, los estudiantes que aprobaron por parciales consideraron que la metodología les sirvió para clarificar y afianzar conocimientos desarrollados en las clases teóricas y los restantes acordaron en que la forma de abordar las preguntas en las CC propició un ambiente de trabajo más agradable para aprender que en otro tipo de clases.

Finalmente, es de destacar que los alumnos que habían aprobado Física I por parciales consideraron a las actividades desarrolladas en las CC como un aporte muy, y bastante, útil respecto de la forma de razonar o analizar situaciones problemáticas. De esto se puede decir que las actividades desarrolladas en estas clases podrían influir positivamente en que los estudiantes puedan internalizar los conocimientos durante el cursado y posibilitar la aprobación de la asignatura por exámenes de término medio.

\section{Agradecimiento}

Este trabajo se llevó a cabo en el marco del proyecto de investigación CAI $+\mathrm{D}$ Código $\mathrm{N}^{\circ}$ : 50120150100122LIsubsidiado por la Universidad Nacional del Litoral, Argentina. 


\section{Referencias}

Ausubel, D., Novak, J. \&Hanesian, H. (1991). Psicología educacional, un punto de vista cognitivo. México: Trillas.

Beatty, I. D.; Gerace, W. J.; Leonard, W. J. and Dufresne, R. J. (2006). Designing effective questions for classroom response system teaching. Am. J. Phys., 74, 31-39.

Budini, N.; Giorgi, S.;Sarmiento, L.; Cámara, C.; Carreri, R.; Marino, L.\&Gómez Carrillo, S. (2016). Implementación de actividades colaborativas en las clases de Física del ciclo inicial universitario, Revista de Enseñanza de la Física, 28 (Extra), 187-195.

Budini, N.; Cámara, C.; Sarmiento, L.; Cámara, C.\&Carreri, R. (2017). Actividades colaborativas sobre conceptos de mecánica en sistemas físicos no puntuales. Revista de Enseñanza de la ciencia, 29 (Extra), 287- 296.

Budini, N.; Marino, L.;Carreri, R; Cámara, C.\&Giorgi, S. (2018). Percepciones de estudiantes luego de implementar "Instrucción entre Pares" en un curso de Física I. Revista de Enseñanza de la ciencia, 30 (Extra), 141- 149.

Byun, T. and Lee, G. (2014). Why students still can't solve physics problems after solving over 2000 problems. Am. J. Phys., 82(9), 906-913.

Calzadilla, M. E. (2002). Aprendizaje colaborativo y tecnologías de la información y la comunicación. Revista Iberoamericana de Educación, 1(10), 1-10.

Cortright, R. N; Collins, H. L. and DiCarlo, S. E. (2005). Peer instruction enhanced student meaningful learning: Ability to solve novel problems. Advances in Physiology Education, 29, 107-111.

Duit, R.; Gropengießer, H.; Kattmann, U.; Komorek, M. \&Parchmann, I. (2012). The model of educational reconstruction - a framework for improving teaching and learning science. En: Jorde, D. and Dillon, J. (Eds.), Science Education Research and Practice in Europe: Retrospective and Prospective, pp. 13-37. Sense Publishers. En: http://www.epitropakisg. gr/grigorise/MER.pdf. Consulta 15/10/2019.

Freeman, S.; Eddy, S. L.; McDonough, M.; Smith, M. K.; Okoroafor, N.; Jordt, H. and Wenderoth, M. P. (2014). Active learning increases student performance in science, engineering, and mathematics. Proc. of the National Academy of Sciences, 23(111), 8410-8415.

Giuliodori, M. J.; Lujan, H. L. and DiCarlo, S. E. (2006). Peer instruction enhanced student performance on qualitative problem-solving questions. Advances in Physiology Education, 30, 168-173.

Martín del Pozo, M.; Basilotta Gómez-Pablos, V. and García-Valcárcel MuñozRepiso, A (2017). A quantitative approach to pre-service primary school teachers' attitudes towards collaborative learning with video games: previous experience with video games can make the difference. International Journal of Educational Technology in Higher Education, 14(1), 11.

Mazur, E. (1997). Peer Instruction. A User's Manual. EstadosUnidos: Prentice Hall.

Meltzer, D. E. and Thornton, R. K. (2012). Resource Letter ALIP-1: ActiveLearning Instruction in Physics. Am. J. Phys., 80(6), 478-496. 
Nicol, D. J. and Boyle, J. T. (2003). Peer instruction versus class-wide discussion in large classes: A comparison of two interaction methods in the wired classroom. Studies in Higher Education, 28(4), 457-473.

Sampieri, R.; Collado, C. \& Lucio, P. (2010). Metodología de la Investigación. México: Mc Graw-Hill Interamericana.

Navío-Marco, J.; Solórzano-García, M. (2019).Student’s social e-reputation ("karma") as motivational factor in MOOC learning. Interactive Learning Environments, 0, 1-15.

Redish, E. F. (2003). Teaching physics: With the physics suite. Hoboken, NJ: John Wiley \& Sons.

Vigotsky, L. (1989). El desarrollo de los procesos psicológicos superiores. Barcelona: Editorial Crítica. 